$34^{\text {th }}$ AIAA Plasmadynamics and Lasers Conference, Orlando, June 2003.

\title{
THE T6 HOLLOW CATHODE: MEASUREMENTS AND MODELING
}

\author{
Mark W. Crofton \\ The Aerospace Corporation, El Segundo, California 90245-4691 \\ and \\ Iain D. Boyd ${ }^{\&}$ \\ University of Michigan, Ann Arbor, Michigan 48109-2140
}

\begin{abstract}
Behavior of the xenon dispenser hollow cathode is known to be complex, exhibiting high through-anode ion emission, unusual particle energy distributions, and high plume erosion rates. A flight-type T6 hollow cathode was characterized using several diagnostic methods. Measurements involved a retarding potential analyzer, quadrupole mass spectrometer, and Langmuir probe. Far-field energy, flux, plasma potential, and charge state distribution data were obtained for plume ions at various operating points. The plume expansion was modeled by explicitly including both electrons and heavy particles in a combined Particle In Cell and direct simulation Monte Carlo approach. The simulations were in good agreement with measured ion energy distributions, which peaked at low energy in comparison to most other studies. Comparisons of ion emission and plasma potential profiles were relatively poor.
\end{abstract}

\section{NOMENCLATURE}

$\begin{array}{ll}\text { A } & \text { ampere } \\ d & \text { keeper-extractor separation } \\ \mathrm{E} & \text { kinetic energy } \\ \mathrm{E}_{\mathrm{f}} & \text { electric field, V/cm } \\ \mathrm{F} & \text { flow rate, } \mathrm{mg} / \mathrm{s} \\ \mathrm{I}_{\mathrm{ck}} & \text { cathode keeper current, Ampere } \\ \mathrm{I}_{\mathrm{F}} & \text { Faraday cup current } \\ \mathrm{I}_{\mathrm{h}} & \text { cathode heater current, Ampere } \\ \mathrm{m} & \text { particle mass, atomic mass units } \\ \mathrm{q} & \text { ion charge }(=\mathrm{ze}) \\ \mathrm{T} & \text { temperature, } \mathrm{K} \\ \mathrm{T}_{\mathrm{i}} & \text { ion temperature, eV } \\ \mathrm{T}_{\mathrm{e}} & \text { electron temperature, eV } \\ \mathrm{V}_{\mathrm{ck}} & \text { cathode keeper potential, referenced to the } \\ & \text { cathode body, Volts } \\ \mathrm{V}_{\mathrm{r}} & \text { repeller potential of the RPA, Volts } \\ \mathrm{Z} & \text { ion charge number } \\ \phi & \text { angle between RPA normal and plume axis } \\ \theta & \text { angle between QMS normal and plume axis }\end{array}$

\section{INTRODUCTION}

Hollow cathodes are of general technological importance. One application is in the area of spacecraft propulsion, where they serve as critical components providing an efficient source of electrons for electric thruster systems. Xenon hollow cathodes can produce single-point failures in gridded ion engine and Hall effect thrusters, and they are an important factor regarding erosion of the screen grid and other components in ion engines. During operation at the high emission current required for high-power ion propulsion systems, the orifice and any components in the plume erode rapidly. ${ }^{1}$ Ions of sufficient energy and flux to cause significant erosion have been observed in plume experiments. ${ }^{2-4}$

Measurements of the ion kinetic-energy distribution in the far-field have been performed on thruster cathodes with a retarding potential analyzer (RPA) or an energy analyzer, ${ }^{2-4}$ in addition to the recent T5 work that utilized a quadrupole mass spectrometer (QMS) and an RPA. ${ }^{5}$ The early results revealed that a significant ion flux was emitted and that the ion energy distribution function could be very broad and peaked at high energy. Ions are abundantly formed and emitted through-anode with energies as much as several times higher than $\mathrm{eV}_{\mathrm{ck}}$ even though the anode potential is above the cathode by $\left|V_{c k}\right|$. Unfortunately, RPA and energy analyzer devices do not distinguish between xenon ions with different charge states if their energy to charge ratios (E/ze) are identical.

The mechanism by which the high-energy ions arise has not been established, but two principal hypotheses have been previously put forward. One mechanism invokes the formation of a potential hill a few $\mathrm{mm}$ downstream from the orifice. ${ }^{3,6}$ Although consistent with some of the data, no clear understanding had emerged of the means by which a hill of sufficient height could be formed. An alternative mechanism has been postulated whereby the current density at the

†Research Scientist, Laboratory Operations/Space Materials Laboratory, M5-754, P.O. Box 92957, Los Angeles, CA 900092957. Member AIAA.

\&Professor, Department of Aerospace Engineering, 1320 Beal Avenue. Associate Fellow, AIAA. 
orifice (on the order of $10^{4} \mathrm{~A} \mathrm{~cm}^{-2}$ ) results in ion acceleration via a magnetohydrodynamic (MHD) effect. $^{2,7}$

A third hypothesis concerning the formation of high-energy ions has been proposed for vacuum arc plasmas, where acceleration of ions also occurs in the direction away from the cathode and E/q can be much greater than the applied voltage. In this case a gas dynamic model is invoked that describes ion acceleration as driven by pressure gradients and electron-ion friction. ${ }^{8,9}$ According to this approach, a small potential hump may exist as a consequence of plasma acceleration rather than as its cause. In the gas dynamic model the ion energy distribution is independent of charge state.

An extensive literature exists on the subject of emitted ion energies from vacuum arcs. The peak plasma densities are an order of magnitude or more higher than applies to hollow cathodes. A second distinguishing characteristic is that the gas supporting the discharge is driven out of the electrodes themselves.

Spatially-resolved experimental measurements of electric potential and the ion velocities near a hollow cathode orifice have previously been performed. ${ }^{10} \mathrm{~A}$ prominent potential hill was not found in either the Langmuir probe or laser-induced fluorescence measurements. The measured velocity distribution near the hollow cathode test articles depended strongly on the cathode type and/or operating point, including plume-mode vs. spot-mode operation.

We have previously studied the T5 cathode, ${ }^{5,11,12}$ a flight-type xenon hollow cathode originally developed for use as the main cathode of a low power ion engine, the UK-10. ${ }^{13}$ The T6 cathode is designed for substantially higher throughput and power input capability, in accordance with its various applications. ${ }^{14}$

As in our previous studies, a retarding potential analyzer (RPA) and a quadrupole mass spectrometer (QMS) were both applied. In addition, Langmuir probe measurements were made in the plume. The computational model developed to perform T5 cathode simulations has now been used to simulate the plume behavior of the T6 cathode, with comparisons presented between model results and experimental data.

The presence of abundant doubly-charged ions was discovered through the QMS measurements. ${ }^{5}$ The higher charge states will contribute significantly to the erosive power of the hollow cathode plume, and be dominant over $\mathrm{Xe}^{+}$at relatively low flux levels. In addition, $\mathrm{Xe}^{2+}$ presence in abundance demonstrated a higher fraction of ionization than had previously been assumed.

QMS measurements of ion energy distribution showed, at least for some T5 operating points, little if any charge state dependence. ${ }^{11}$ This result provided evidence for the potential hill model. The fact that large current increases at a given flow rate did not correlate well with high-energy, broad distributions did not support the MHD hypothesis.

Most previous hollow cathode characterization studies have incorporated a large anode shell and a secondary discharge between the cathode and this anode to simulate the ion engine environment. Such an approach complicates the study of the hollow cathode plume. The hollow cathode was operated in a standalone configuration in the present study. Recent evidence has suggested that the difference between the two configurations can be dramatically different in terms of emitted ion energy distributions. ${ }^{15}$

As a thruster component hollow cathodes are much simpler to build and operate than the systems in which they are incorporated. Hollow cathodes will make attractive stand-alone ion thrusters if the ion emission current and device efficiency can become more comparable to existing ion thruster systems. Improved understanding of hollow cathode behavior will aid in its development as an optimized thruster component and potentially as a stand-alone ion thruster.

\section{EXPERIMENTAL}

A xenon hollow cathode was installed in a 75-cmdiameter vacuum chamber, pumped by a $1000 \mathrm{l} / \mathrm{s}$ (on nitrogen) turbomolecular pump and a 12,500/4,500/ 1,000 l/s (hydrogen/water/xenon) TMP150 cryopump (CVI) mounted on a 10-in. conflat flange. The cryopump could be readily isolated from the chamber by an 8 in. electropneumatic gate valve. The base pressure with no xenon flow was about $2 \times 10^{-7}$ Torr. At the maximum xenon flow rate of $1.08 \mathrm{mg} / \mathrm{s}$, the background pressure indicated by an ion gauge positioned far from the cryopump was $1.1 \times 10^{-4}$ Torr (cathode off), after applying a standard sensitivity correction for xenon. The ion gauge was located well above and behind the hollow cathode orifice, at a distance of more than $40 \mathrm{~cm}$.

The cathode was installed on a rotatable table, with a QMS positioned behind a fixed beam skimmer, and the RPA also viewing the plume near the orifice (see Fig. 1). The range of rotary motion for the hollow cathode was $\theta=-50$ to $+135 \mathrm{deg}$. (measured with respect to the axis of the QMS). The rotary table was mounted on a linear stage with $12 \mathrm{~cm}$ of free translation range along the perpendicular to the skimmer axis. The $5-\mathrm{mm}$ aperture of the grounded skimmer was about 50 $\mathrm{mm}$ downstream from the keeper orifice. The entrance of the QMS was about $50 \mathrm{~cm}$ further downstream, and aligned with the hollow cathode orifice and beam skimmer. Differential pumping was utilized to lower the background pressure in the region between QMS and skimmer. In the present study, the QMS was always operated with detector on and ionizer turned off. 
The RPA consisted of a Faraday cup (Kimball Physics, FC-72A) with four closely spaced grids at the input. The entrance and third grid were grounded, and the fourth or innermost was biased negative to reject plasma electrons and to suppress the loss of secondary electrons. The second grid operated at the retarding potential, $\mathrm{V}_{\mathrm{r}}$. The acceptance area of the entrance aperture was $1.0 \mathrm{~cm}^{2}$, and each of the grid apertures was covered with a fine tungsten mesh. The aperture was located $21 \mathrm{~cm}$ from the cathode orifice.

To check for differences of ion energy distribution with charge state, a variable potential was applied to a retarding electrode positioned just downstream from the grounded exit plane of the skimmer. By tuning the QMS to the transmission peak for a particular charge state, approximate RPA data could be obtained with detection of a single ion species.

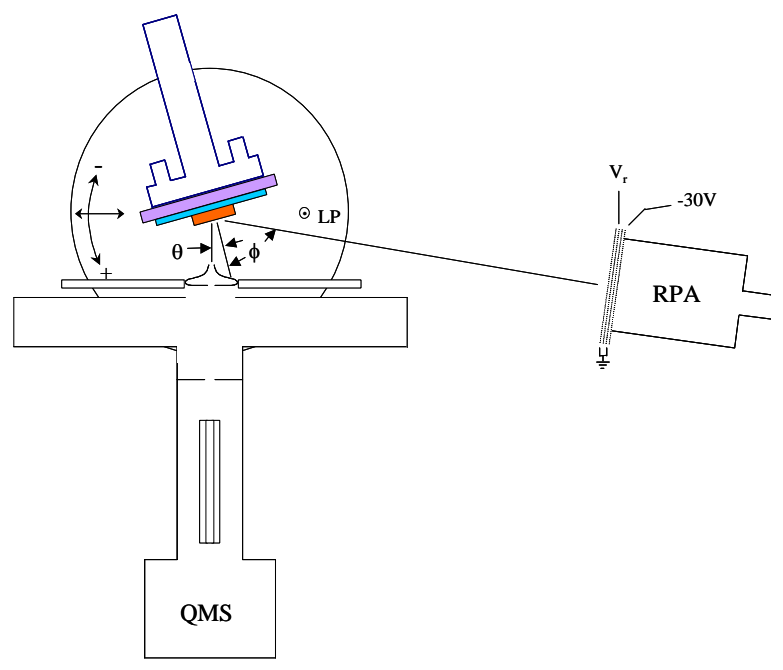

Figure 1. Schematic of the experimental setup, top view.

A Langmuir probe constructed with 1-mm tungsten wire and shielded with ceramic insulator was mounted vertically in the plume. The probe was placed at $\theta=90 \mathrm{deg}$. The linear translation stage was used to vary the axial cathode to probe distance. A 2-mm length of the tungsten wire was exposed to the plume.

The hollow cathode contained an impregnated tungsten dispenser with an inside diameter of $2.0 \mathrm{~mm}$, outside diameter of $5.0 \mathrm{~mm}$, and length of $20 \mathrm{~mm}$, which acts as a chemical factory to release barium to the surface at an appropriate rate to achieve low work function and long life. The dispenser must be at approximately $1000{ }^{\circ} \mathrm{C}$ for the cathode to operate normally. The orifice of the hollow cathode, machined out of solid tantalum, was $0.9 \mathrm{~mm}$ in diameter $\times 1.0$ $\mathrm{mm}$ long, with a downstream half-angle chamfer of 45 degrees (see Fig. 2). The cross-sectional area of the T6 orifice in comparison to the T5 was $20 \times$ greater. A keeper electrode with 5-mm-diameter aperture was positioned just downstream from the nozzle in an enclosed configuration. A fraction of the xenon flow can escape on the periphery. The hollow cathode had not

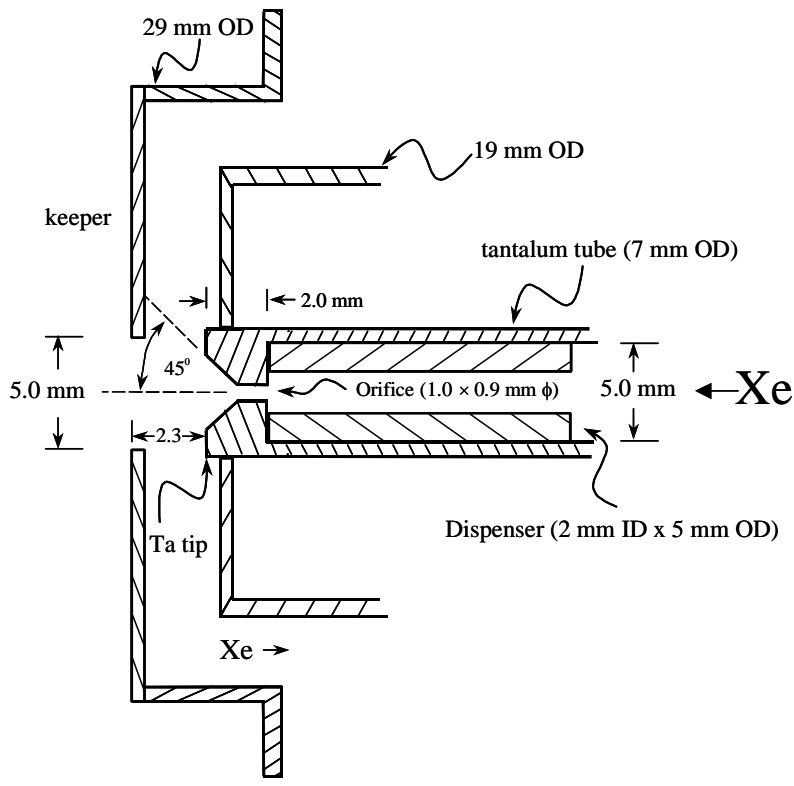

Figure 2. Schematic cross section of the nozzle/keeper configuration, approximately to-scale. The heater, radiation shield, and other items are not shown.

been operated previously. For the present study the device was run at a number of different operating points (see Table 1).

The cathode to anode voltage, $\mathrm{V}_{\mathrm{ck}}$, was always under $30 \mathrm{~V} . \mathrm{V}_{\mathrm{ck}}$ depends on the cathode temperature, flow rate, $I_{c k}$ (which influences cathode temperature), and presumably the condition of the dispenser, size of the orifice and other details of the geometry. At high flow rate and temperature, such as may be obtained after startup before the cathode heater is shutdown, $\mathrm{V}_{\mathrm{ck}}$ can be below $10 \mathrm{~V}$. The cathode discharge current was never set higher than $20 \mathrm{~A}$ in this study.

Spot mode operation was not observed in this study, and would be difficult to produce due to the chamfered orifice. Each operating point is believed to have been a plume mode, although beam mode operation is also possible. ${ }^{7}$

\section{MODEL DESCRIPTION}

Models of hollow cathode plumes have been developed by Parks et $\mathrm{al}^{16}$ and by Williams and Wilbur. ${ }^{17}$ In Ref. 16, a fluid model of the electrons combined with an assumed profile for the ion density was used to model a mercury hollow cathode. By 
assuming anomalously low electrical conductivity (reduced by a factor of about 1000), good agreement was obtained for measurements of plasma potential and electron temperature.

The model under development in this work seeks to go beyond that described in Ref. 16 by explicitly modeling both the electrons and the heavy particles (ions and neutrals). Due to the low density nature of the hollow cathode plumes, a kinetic, particle approach is employed to simulate the xenon ions and neutral atoms. A detailed fluid model of the electrons is also employed.

The ions and neutrals are treated using a combination of the Particle In Cell method (PIC) ${ }^{18}$ for transporting the ions in electrostatic fields, and the direct simulation Monte Carlo method (DSMC) ${ }^{19}$ for performing collisions and transporting the neutral atoms. Momentum transfer and charge exchange collisions are the only collision mechanisms implemented at this stage.

The spatial distribution of ions gives the electron number density under the assumption of charge neutrality. Solution of the electron continuity equation then provides the electron velocity, $\mathbf{v}_{\mathrm{e}}$. The electron momentum equation is given by: ${ }^{20}$

$$
\frac{\partial}{\partial t}\left(m_{e} n_{e} \mathbf{v}_{e}\right)+m_{e} n_{e}\left(\mathbf{v}_{e} \cdot \nabla\right) \mathbf{v}_{e}=-e n_{e} \mathbf{E}-\nabla p_{e}+\mathbf{R}
$$

where $m_{e}$ is the mass of an electron, $n_{e}$ is the electron number density, $v_{\mathrm{e}}$ is the electron velocity vector, $\mathbf{E}$ is the electric field, $\mathrm{p}_{\mathrm{e}}$ is the electron pressure, and $\mathbf{R}$ is the friction term. It is further assumed that the electrons behave as a perfect gas $\left(\mathrm{p}_{\mathrm{e}}=\mathrm{n}_{\mathrm{e}} \mathrm{kT}_{\mathrm{e}}\right)$, and that the friction term is given by:

$$
\mathbf{R}=\frac{e n_{e} \mathbf{j}}{\sigma}
$$

where $\mathbf{j}$ is the current density, and $\sigma$ is the electrical conductivity.

Assuming a steady state, neglecting the inertial term on the left hand side of Eq. (1), and introducing the plasma potential $-\nabla \phi=\mathbf{E}$, a generalized Ohm's law is obtained:

$$
\left.\mathbf{j}=\sigma-\nabla \phi+\frac{1}{e n_{e}} \nabla\left(n_{e} k T_{e}\right)\right\rfloor
$$

The charge continuity condition with source term $\mathrm{S}_{\mathrm{e}}$ due to ionization:

$$
\nabla \cdot \mathbf{j}=S_{e}
$$

is then solved to obtain the plasma potential. This equation is written as a Laplace equation with weak source terms and is solved using an Alternating Direction Implicit (ADI) scheme.

The electron energy equation is given by: ${ }^{20}$

$$
\begin{aligned}
& \frac{\partial}{\partial t}\left(\frac{3}{2} n_{e} k T_{e}\right)+\frac{3}{2} n_{e}\left(\mathbf{v}_{e} \cdot \nabla\right) k T_{e}+p_{e} \nabla \cdot \mathbf{v}_{e}= \\
& \nabla \cdot \kappa_{e} \nabla T_{e}+\mathbf{j} \cdot \mathbf{E}-3 \frac{m_{e}}{m_{i}} v_{e} n_{e} k\left(T_{e}-T_{H}\right)-n_{e} n_{a} C_{i} \varepsilon_{i}
\end{aligned}
$$

where $m_{i}$ is the ion mass, $v_{e}$ is the total electron collision frequency, $\kappa_{\mathrm{e}}$ is the electron thermal conductivity, and $\mathrm{T}_{\mathrm{H}}$ is the heavy particle temperature. Again assuming a steady state, and neglecting the collision term as small:

$$
\begin{aligned}
& \nabla^{2} T_{e}=-\frac{1}{\kappa_{e}} \nabla \cdot \kappa_{e} \nabla T_{e}+\frac{1}{\kappa_{e}}\left\{-\mathbf{j} \cdot \mathbf{E}+\frac{3}{2} n_{e}\left(\mathbf{v}_{e} \cdot \nabla\right) k T_{e}\right. \\
& \left.+p_{e} \nabla \cdot \mathbf{v}_{e}+3 \frac{m_{e}}{m_{i}} \nu_{e} n_{e} k\left(T_{e}-T_{H}\right)+n_{e} n_{a} C_{i} \varepsilon_{i}\right\}
\end{aligned}
$$

where $\mathbf{j}$ is obtained from Eq. (3) after the plasma potential is calculated. Equation (6) is again a Laplace equation with weak source terms that is solved using the ADI approach. The transport coefficients are evaluated using the basic definitions from Ref. 20:

$$
\begin{gathered}
\sigma=\frac{e^{2} n_{e}}{m_{e} v_{e}} \\
\kappa_{e}=\frac{2.4}{1+\frac{v_{e i}}{\sqrt{2} v_{e}}} \frac{k^{2} n_{e} T_{e}}{m_{e} v_{e}}
\end{gathered}
$$

where $v_{\mathrm{e}}=v_{\mathrm{ei}}+v_{\mathrm{en}}, v_{\mathrm{ei}}$ is the ion-electron collision frequency, $v_{\mathrm{en}}$ is the neutral-electron collision frequency, and these frequencies are evaluated for the xenon system using cross sections provided in Ref. 20.

The model calculations begin at the exit of the orifice nozzle. The flow conditions are estimated from the measured mass flow rate and current in addition to making assumptions for the species temperatures. In the present work, an electron/ion temperature of 1.5 to 2.0 $\mathrm{eV}$ is employed while the neutrals are assumed to be at the cathode temperature of $1300 \mathrm{~K}$. The plasma density obtained in this manner is consistent with a detailed model of the hollow cathode insert and orifice regions developed by Domonkos. ${ }^{21}$

Boundary conditions are also required for plasma potential and electron temperature in the solutions of the current conservation (Eq. (4)) and electron energy (Eq. (6)) equations. The potential of the keeper is set along the surfaces of the keeper and the flow inlet, and zero-gradient conditions are employed along all other boundaries. For electron temperature, an isothermal 
condition is used along the inlet plane and zero-gradient conditions are applied everywhere else.

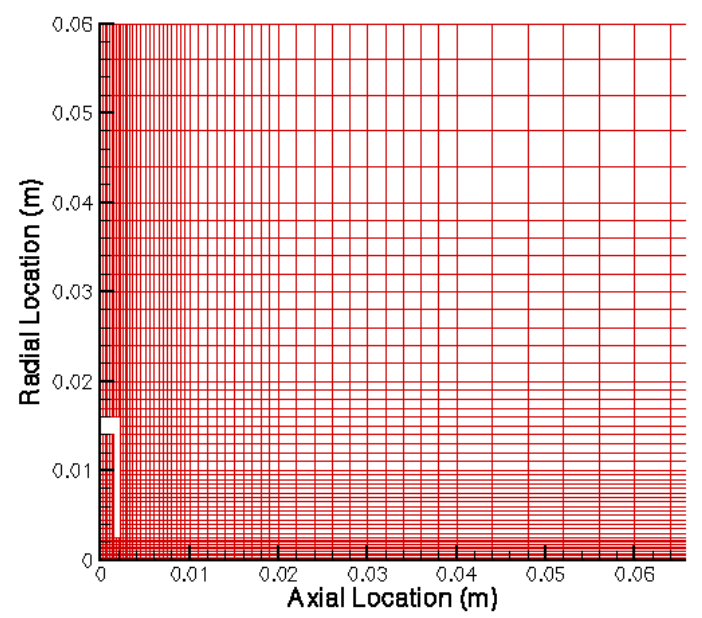

Figure 3. Detail of computational grid showing the orificekeeper and plume regions.

The computational domain extends to $0.30 \mathrm{~m}$ along the axis from the orifice and $0.25 \mathrm{~m}$ radially from the centerline. A portion of the grid is shown in Fig. 3 and employs 110 by 90 non-uniform, rectangular cells. A time-step of $5 \times 10^{-9} \mathrm{~s}$ is employed, which is smaller than the inverse plasma frequency. The simulation typically reaches a steady state after about 25,000 iterations and final results are obtained by averaging over a further 20,000 iterations. About 500,000 particles are employed in each simulation.

The simulations are primarily performed for operating points 4 and 10. Assessment of the results is performed through comparisons with the measured data for current profile and ion energy distribution obtained with the RPA instrument. These data are recorded during the simulation at the same location as in the RPA experiments (about $21 \mathrm{~cm}$ from the cathode).

\section{RESULTS AND DISCUSSION}

\section{Measured Results}

In our previous study of the T5 cathode, test chamber pressure variation following a significant change in cathode operating point was consistent with a variable nozzle flow impedance. Assuming thermal equilibrium with the vacuum chamber walls and given that the flow system maintains a constant mass flow rate into the cathode reservoir, the observed instantaneous drop in chamber pressure upon switching on the T5 discharge was proposed to arise from a corresponding increase of flow impedance. This impedance could arise from two mass transport effects: the upstream migration of ions through the orifice, driven by the local electric field, and collisional momentum transfer to neutrals by the upstreaming ions. In a recent PIC-DSMC simulation of the T6 cathode interior, theoretical support was provided for these mass transport mechanisms. ${ }^{22}$

The test chamber pressure is proportional to the mass flow rate exhausted by the cathode. According to this measure, the expellant flow rate of the T5 changed abruptly following a keeper current adjustment, and returned slowly to its original equilibrium value. A change in the T5 heater current during the startup cycle required a similar period of time to return to equilibrium, however the change was always gradual in this case, as may be expected from a thermal phenomenon. The chamber pressure would initially fall, possibly because the pressure elevation in the xenon gas behind the nozzle is constrained by the pressure in the cold gas reservoir of the supply line. With the metering system supplying constant flow into the reservoir, eventually the nozzle backing pressure must rise enough to reproduce the original nozzle flow rate prior to the perturbation. If the nozzle backing pressure changes slowly compared to the temperature (due to the large size of the reservoir and low flow rate), an initial $\mathrm{T}^{-1 / 2}$ dependence in the test chamber pressure would be expected, where $\mathrm{T}$ is the stagnation temperature. ${ }^{23}$

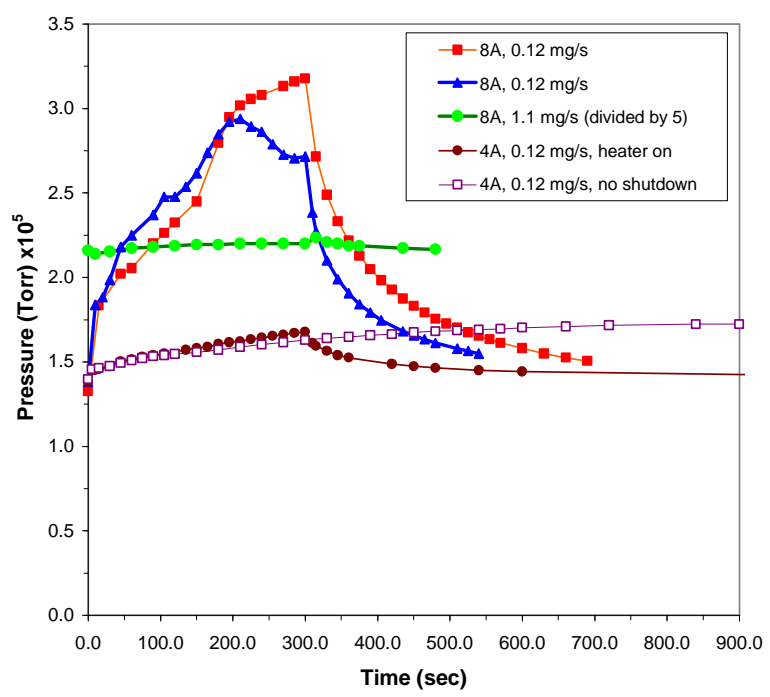

Figure 4. Test chamber pressure variation following turn-on of the T6 keeper discharge, for operating points shown. In most cases the discharge was switched off again after 300 seconds.

When the T5 keeper discharge was switched on or off, the change in the background pressure of the vacuum chamber was large and immediate, followed by a slow return to the original level, on a time scale of the order of 10 minutes. This observation indicated that the initial effect was nonthermal. The subsequent drift toward equilibrium and return to the starting chamber pressure 
could be thermal or determined by the flow rate and rate of change of nozzle backing pressure.

Behavior of the vacuum chamber background pressure was very different for the T6 cathode. Changes of keeper current levels did not produce

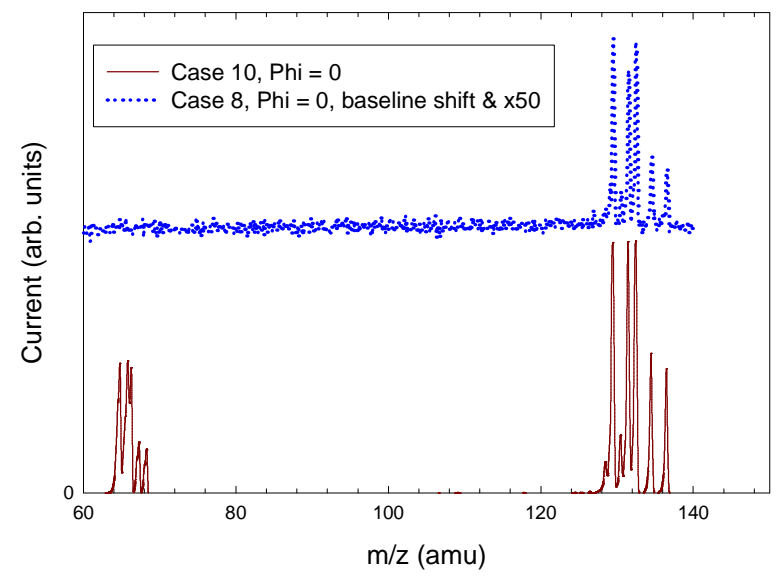

Figure 5. Mass spectral scans for two cases, showing the contrast in $\mathrm{Xe}^{2+} / \mathrm{Xe}^{+}$ratio.

the large, abrupt changes observed with the T5. Figure 4 illustrates the T6 results. Initial changes of background pressure were of opposite sign to the T5 case and varied little at high flow settings, especially if $\mathrm{I}_{\mathrm{ck}}$ was not set high. The timescale for variations that did occur was on the order of 10 minutes. Exponential decay of the pressure was observed following discharge shutdown, without a step function at turn-off. However, the case of high current and low flow rate $\left(\mathrm{I}_{\mathrm{ck}}=8.0 \mathrm{~A}, \mathrm{~F}\right.$ $=0.12 \mathrm{mg} / \mathrm{s}$ ) exhibited a large, slow rise that was neither smooth nor closely repeatable.

Previous measurements on a T6 hollow cathode have revealed a complex variation of its back (internal) pressure with current and flow levels. ${ }^{7}$ A mechanism involving the $\mathbf{j} \times \mathbf{B}$ force and $\mathrm{a} \mathrm{z}$ pinch effect was proposed as the cause of the elevated back pressure. The $\mathrm{z}$ pinch mechanism presumes a runaway increase in the $\mathbf{j} \times \mathbf{B}$ force below some critical flow rate or orifice pressure, ${ }^{7}$ and may provide an alternate or supplemental mechanism to explain the cathode behavior.

The hypothetical flow impedance proposed in the T5 case is not able to explain the T6 data because the background pressure changes are of opposite sign and the chamber pressure did not return to the starting point within a reasonable timeframe. Flow impedance effects can be much smaller under most of the conditions of this study, as much as $400 \times$ lower for case 1 vs. a typical T5 operating point with the same flow rate because the T6 cross-sectional area is $20 \times$ greater. The most likely explanation for the T6 data seems to be elevation of vacuum chamber outgassing rates when the cathode discharge is on. A heat lamp inside the chamber was able to produce a similar effect when switched on and off.

In our previous study of the T5 hollow cathode, ${ }^{5}$ the mass spectrometer indicated a rapid initial rise in both $\mathrm{Xe}^{2+}$ and $\mathrm{Xe}^{+}$flux with increasing keeper current. A similar result was obtained with the T6, and the variability of $\mathrm{Xe}^{2+}: \mathrm{Xe}^{+}$ratio is illustrated by Fig. 5 . The two cases shown have identical flow rates, but the $\mathrm{Xe}^{2+}: \mathrm{Xe}^{+}$ratio is much higher at $\mathrm{I}_{\mathrm{ck}}=20 \mathrm{~A}$ than at $8 \mathrm{~A}$. Similar results were obtained at other operating points, with $\mathrm{I}_{\mathrm{ck}}$ levels scaled according to the flow rate.

Ion current emitted from the T6 hollow cathode was collected as a function of the RPA repeller voltage. The data of Fig. 6 were obtained for several operating points (see Table 1). These data indicate, as expected, that collected Faraday cup current rises with discharge current. The flow rate is a more influential parameter than keeper current in determining the RPA profile. At the $0.50 \mathrm{mg} / \mathrm{s}$ flow rate, the main effect of increasing $I_{c k}$ is to push up the starting point of the RPA profile while largely preserving its shape. The current levels at $V_{r}=0$ obviously have a nonlinear dependence on $\mathrm{I}_{\mathrm{ck}}$, a result that is especially obvious in comparing the low-flow case to the others. For the 0.125 and $0.50 \mathrm{mg} / \mathrm{s}$ cases with 4.0A keeper current, the low-flow (case 2) RPA current was much greater as expected because of its relatively high keeper voltage. The low-flow RPA profile is also distinguished by a long tail and a transition region that occurs at relatively high $\mathrm{V}_{\mathrm{r}}$.

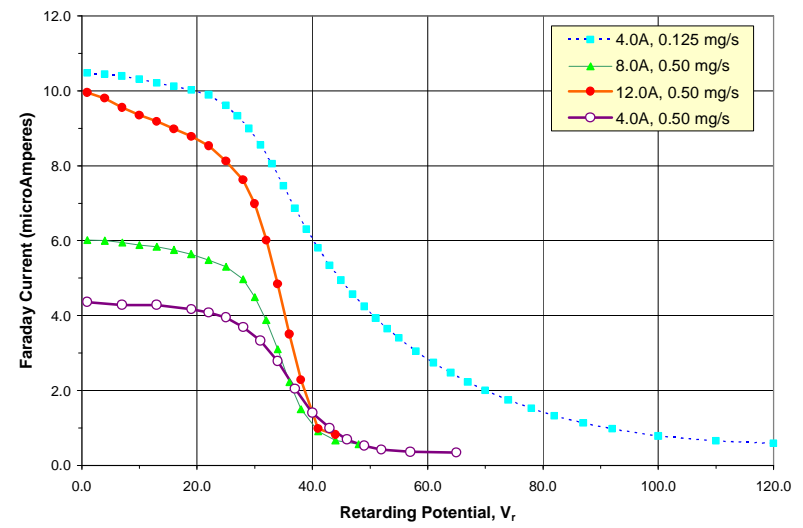

Figure 6. RPA current as a function of repeller voltage at various operating points.

The quantity $\Delta \mathrm{I}_{\mathrm{F}} / \Delta \mathrm{V}_{\mathrm{r}}$ approximates the ion energy distribution. When each ion energy distribution function (IEDF) is computed from the RPA profiles of Fig. 6, the $0.125 \mathrm{mg} / \mathrm{s}$ operating point stands out from the others. While the IEDF width decreased slightly with increasing current for $\mathrm{F}=0.50 \mathrm{mg} / \mathrm{s}$, it broadened dramatically when the flow was reduced to $0.125 \mathrm{mg} / \mathrm{s}$ (see Fig. 7). With $30 \%$ higher $\mathrm{V}_{\mathrm{ck}}$ and one-fourth the 
gas density, a substantially elevated reduced field, $\mathrm{E}_{\mathrm{f}} / \mathrm{n}$, will exist at this operating point. The rise in reduced field may produce higher electron and ion temperatures and a broadened ion energy distribution. ${ }^{12,24}$ The fullwidth-half-maximum of the distribution is about $25 \mathrm{~V}$ for case 2, and much less for the other cases shown. A significant number of ions with E/q exceeding $100 \mathrm{~V}$ are present. For most of the T6 cases shown in Fig. 7 the width of the distribution is considerably less than those obtained in the T5 study. ${ }^{11}$ In the T5 simulations the most influential parameter regarding the IEDF was the electron temperature. In contrast, at a single flow setting the value of $\mathrm{I}_{\mathrm{ck}}$ has only a minor effect on $\mathrm{V}_{\mathrm{ck}}$ and the IEDF width.

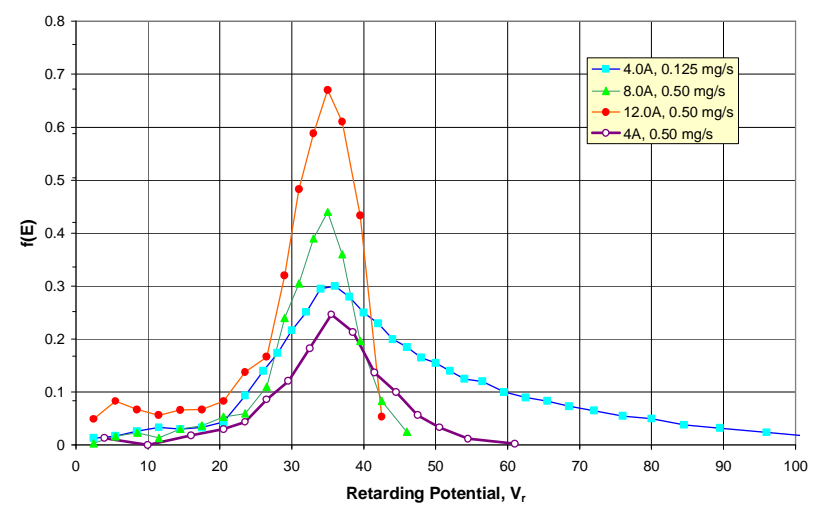

Figure 7. Ion Energy Distribution Functions for selected operating points at normal incidence; $f(E)=-\Delta I_{F} / \Delta V_{r}$.

RPA profiles for various operating points with $\mathrm{F}=$ $1.08 \mathrm{mg} / \mathrm{s}$ are plotted in Fig. 8. The relatively low peak energy of the $\phi=90 \mathrm{deg}$. distribution is consistent with charge exchange formation or large angle ion scattering off neutral xenon. At small $\phi$ the ions are more energetic. This was also the case in the T5 study. As in that study, low flow rate and high discharge voltage are associated with relatively high-energy distributions.

The profile at $\mathrm{I}_{\mathrm{ck}}=20 \mathrm{~A}$ was found to slowly shift toward lower energy. The "warming up" and "fully warmed up" profiles in Figs. 8 and 9 were obtained several hours apart. The shape of the latter profile is anomalous, as $I_{F}$ rises ahead of the steep transition region. No significant difference was observed in respective operating parameters despite the large shift in peak energy.

RPA profiles and ion energy distributions are shown in Figures 10 and 11, respectively, for a single operating point that is approximately case $4\left(\mathrm{~V}_{\mathrm{ck}}=\right.$ 17.8; the keeper potential was significantly lower upon returning to this operating point for further study).

Langmuir probe data obtained under case 4 conditions are plotted in Fig. 12. Plasma potential, floating potential, and electron temperature all decrease significantly in the downstream direction. A similar set of data was obtained for case 2, with slightly lower plasma potential and higher $\mathrm{T}_{\mathrm{e}}$.

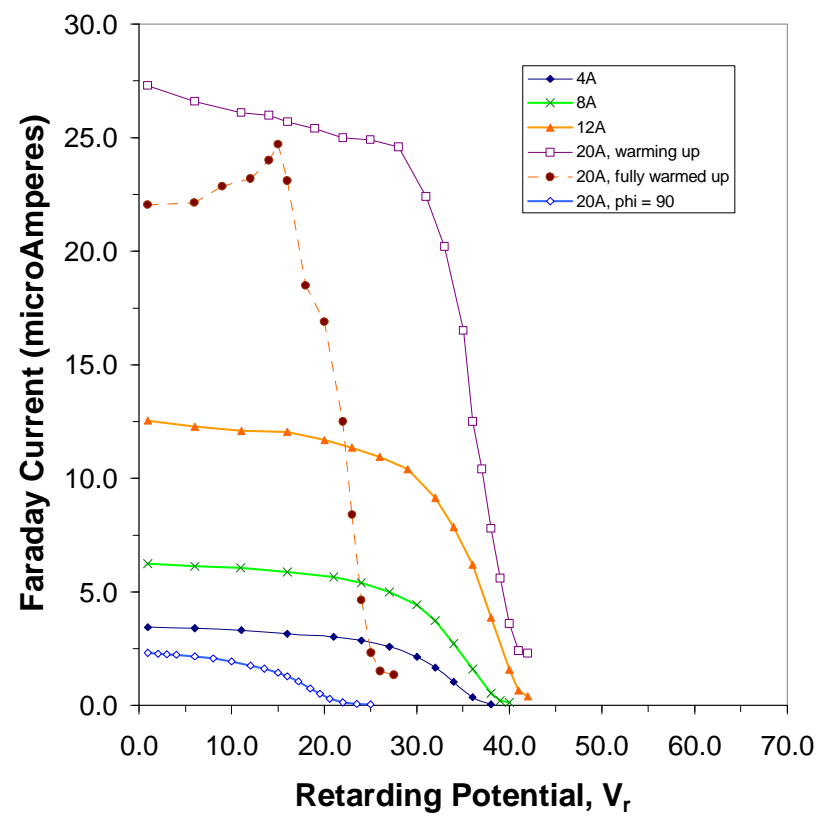

Figure 8. RPA current as a function of repeller voltage at various operating points, $\mathrm{F}=1.08 \mathrm{mg} / \mathrm{s}$.

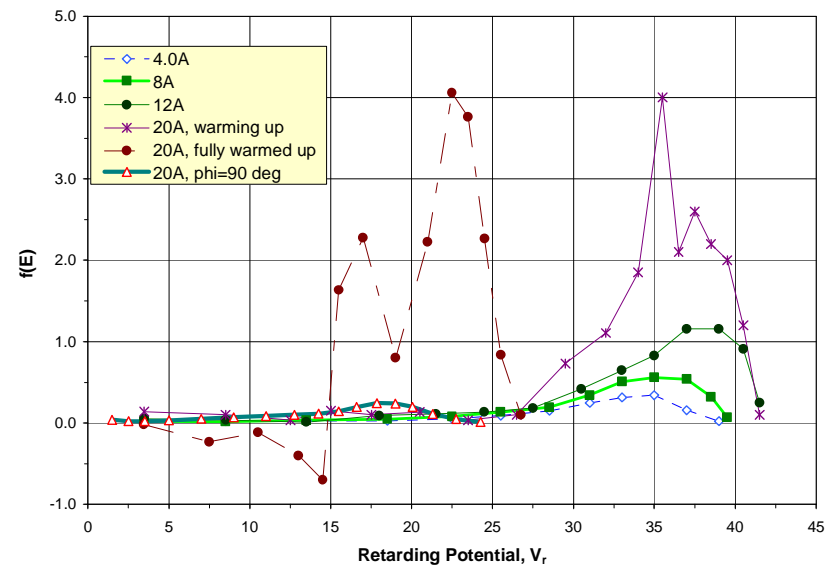

Figure 9. Ion Energy Distribution Functions for selected operating points at normal incidence, except where indicated; $\mathrm{f}(\mathrm{E})=-\Delta \mathrm{I}_{\mathrm{F}} / \Delta \mathrm{V}_{\mathrm{r}}$.

\section{Comparison of Simulated and Measured Results}

Comparison of plasma potential and electron temperatures for case 2 show that the measured $T_{e}$ exhibits greater variation and the potential less variation than the simulation (see Fig. 13). The rapid change in plasma potential predicted by the model was not observed in the measurement. If a high potential gradient exists near the keeper, it evidently extends less than $3 \mathrm{~mm}$ out from the keeper exit plane. 
In Figs. 14-16 comparisons are shown between simulation and experiment for case 2, 4, and 10 IED functions, respectively, on the centerline $(\phi=0)$. Excellent agreement was obtained for both width and peak position in each case, with $\mathrm{T}_{\mathrm{e}}=1.5 \mathrm{eV}$ for case 4 and $\mathrm{T}_{\mathrm{e}}=2.0 \mathrm{eV}$ for cases 2 and 10 . The most significant discrepancy is that the simulation did not reproduce the high-energy tail of the experimental distribution for case 2. A similar comparison for case 10 with $\phi=90 \mathrm{deg}$. and the same $\mathrm{Te}$ as $\phi=0 \mathrm{deg}$., also did very well (see Fig. 17).

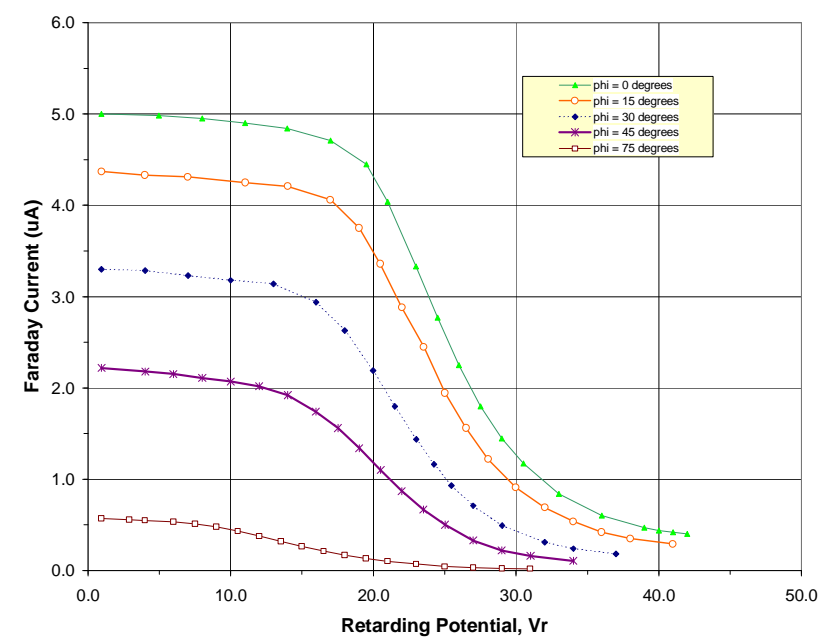

Figure 10. RPA current as a function of repeller voltage at various viewing angles, $\sim$ case $4\left(\mathrm{~V}_{\mathrm{ck}}=17.8\right)$.

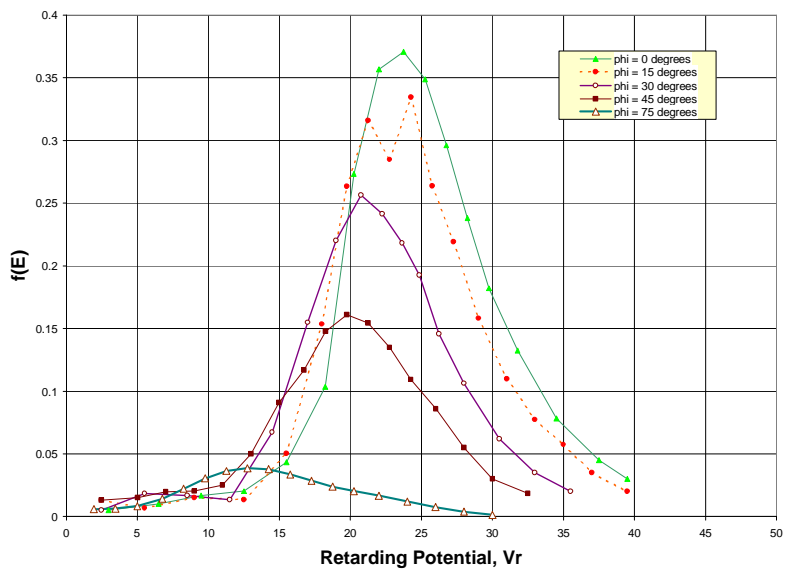

Figure 11. Ion Energy Distribution Functions, $\sim$ case $4\left(\mathrm{~V}_{\mathrm{ck}}=\right.$ $17.8)$ at various RPA viewing angles; $f(E)=-\Delta \mathrm{I}_{\mathrm{F}} / \Delta \mathrm{V}_{\mathrm{r}}$.

Comparisons of simulated vs. measured angular ion emission profiles for cases 10 and 4 (see Figs. 18 and 19 , respectively) reveal order of magnitude discrepancies. The simulation over-predicts the emission flux, and the experimental profile for case 10

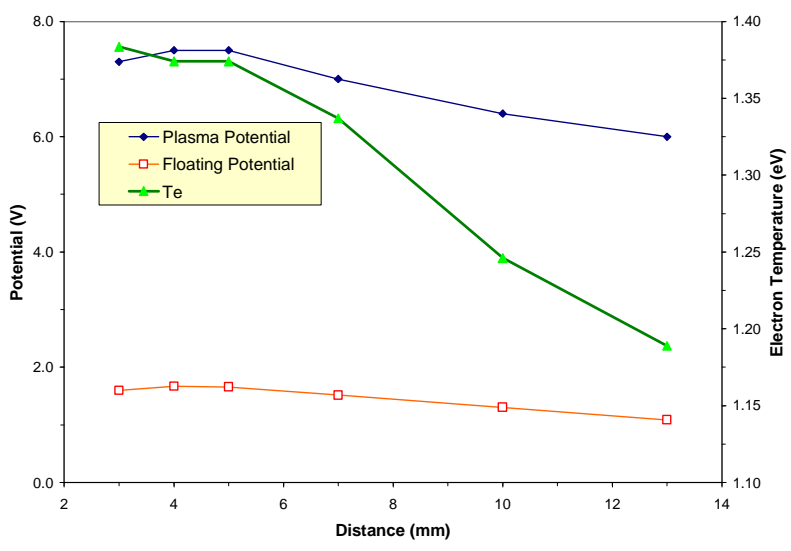

Figure 12. Summary of measured Langmuir probe results for case 4 . The distance was measured from probe center to the downstream keeper face.

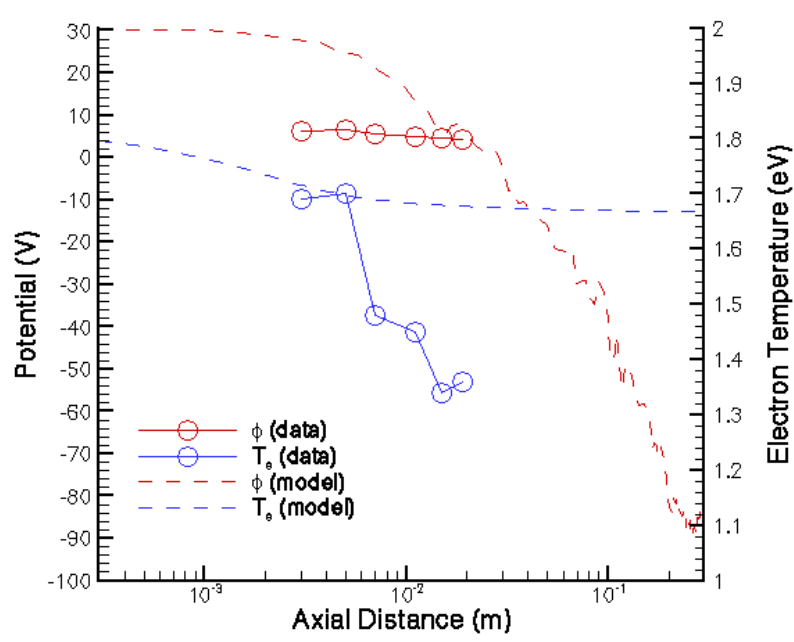

Figure13. Comparison of measured and simulated plasma potential and electron temperature profiles, case 2 .

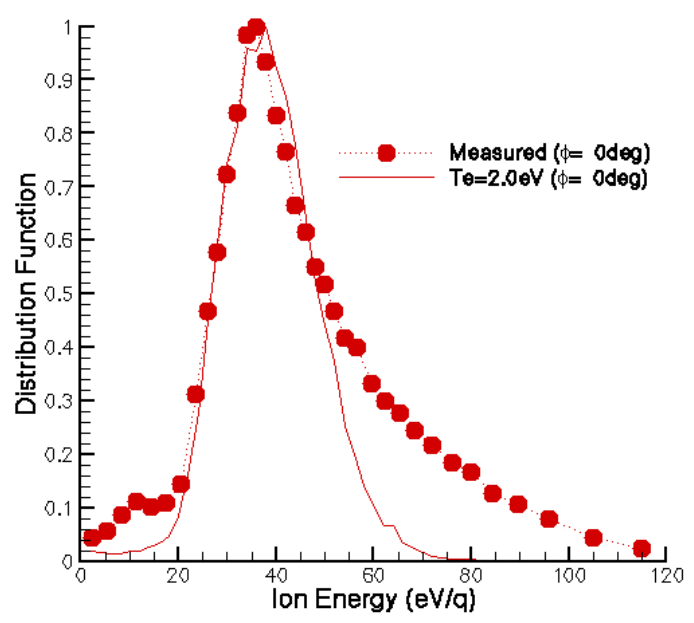

Figure 14. Ion energy distributions at the RPA position for case $2\left(I_{\mathrm{ck}}=4.0 \mathrm{~A}, \mathrm{~F}=0.125 \mathrm{mg} / \mathrm{s}\right), \phi=0 \mathrm{deg}$. 
is obviously more flat than its theoretical counterpart. The shapes of the profiles compare better for case 4 .

\section{Discussion}

The only parameter adjusted in the T6 simulations to-date was $\mathrm{T}_{\mathrm{e}}$. The $\mathrm{T} 5$ simulations showed that $\mathrm{T}_{\mathrm{e}}$ and $v_{\text {ei }}$ exerted the most influence on the results. The simulation is also sensitive to assumptions concerning plasma potential distribution near the keeper aperture. While further parameter adjustments may improve the agreement between simulation and experiment, additional probe data in the orifice-keeper region is clearly needed to determine the correct parameters in this critical region and to establish definitive boundary conditions.

In the potential hill model, energy distributions will depend strongly on charge state $(\mathrm{E} \sim \mathrm{qV})$ and

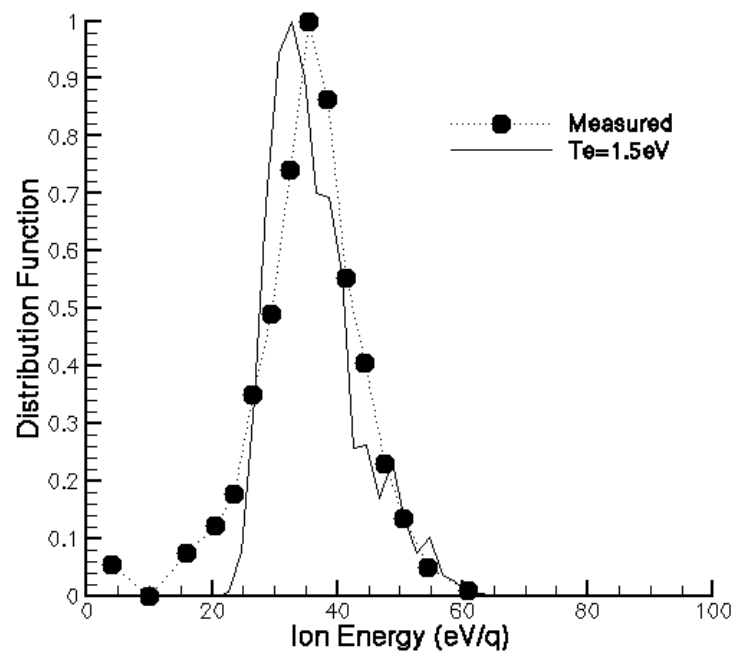

Figure 15. Ion energy distributions at the RPA position for case $4\left(\mathrm{I}_{\mathrm{ck}}=4.0 \mathrm{~A}, \mathrm{~F}=0.50 \mathrm{mg} / \mathrm{s}\right), \phi=0 \mathrm{deg}$.

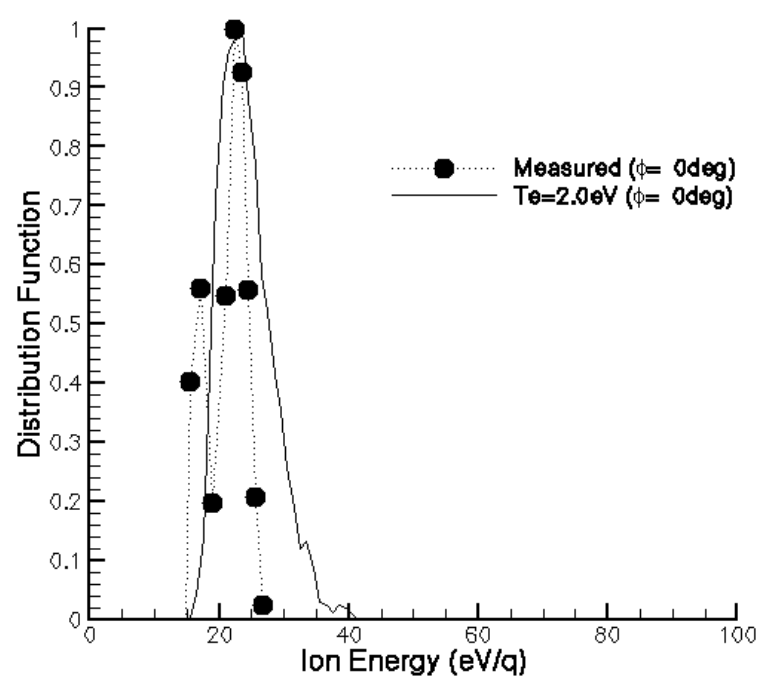

Figure 16. Ion energy distributions at the RPA position for case $10\left(I_{\mathrm{ck}}=20.0 \mathrm{~A}, \mathrm{~F}=1.08 \mathrm{mg} / \mathrm{s}\right), \phi=0 \mathrm{deg}$. largely coincide when plotted vs. $\mathrm{V}_{\mathrm{r}}\left(\mathrm{V}_{\mathrm{r}}=\mathrm{E} / \mathrm{q}\right)$. In contrast, the gas dynamic model predicts the kinetic energy distributions will be similar for all ions. ${ }^{8,25,26}$ Available T5 and T6 cathode data imply that the kinetic energy distributions for $\mathrm{Xe}^{+}$and $\mathrm{Xe}^{2+}$ are similar. The potential hill model gives primary importance to plasma potential and electron temperature, whereas in the gas dynamic model the electron-ion friction term and ionelectron collision rates are critical. The simulation suggests that all of these parameters may contribute, however $\mathrm{T}_{\mathrm{e}}$ dominates in the regime investigated. It should also be noted that individual terms in the electron energy equation usually contain more than one parameter (see Equ. 6), and $\mathrm{T}_{\mathrm{e}}$ appears multiple times.

The IED peaks appeared at quite low energy in this study, similar to a previous result obtained with the T6 cathode placed in a simulated thruster discharge chamber. ${ }^{7}$ Recent electrostatic analyzer measurements with a different cathode in a well-simulated thruster discharge chamber found many ions at high energy, and very strong dependence of the distribution on flow rate, current, and line-of-sight, with relatively low energy ions seen when viewing the orifice on-axis. ${ }^{15}$ In the present study, however, the highest IEDF is obtained when viewing the orifice on-axis. It is not clear whether the T6 cathode has a high propensity to produce low energy ions (good for thruster applications as an electron source, bad for standalone micro-thrust applications) under typical operating conditions, or whether this is a matter of appropriately simulating the thruster discharge chamber. It is to be expected, however, that the influence of the ion-thruster chamber and its discharge will very significantly influence the energy spectrum of detected ions, due to the high discharge current, magnetic field, and containment

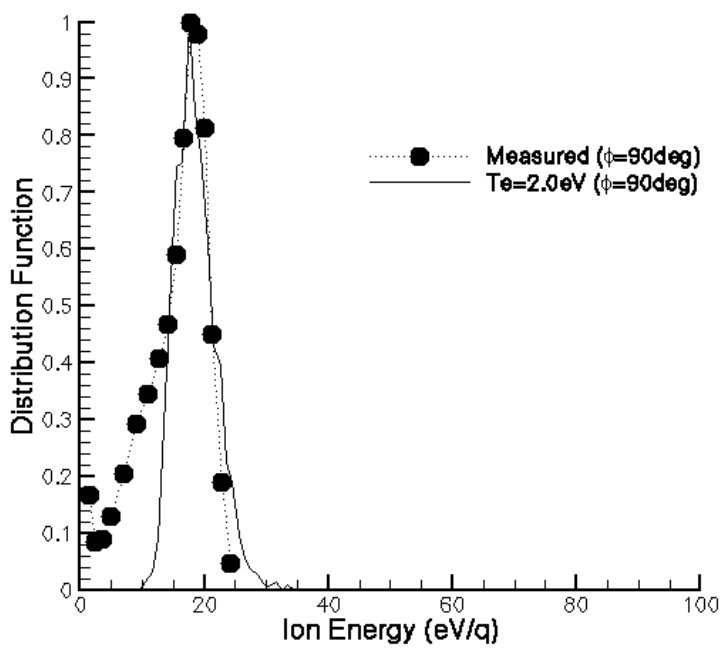

Figure 17. Ion energy distributions at the RPA position for case $10\left(I_{\mathrm{ck}}=20.0 \mathrm{~A}, \mathrm{~F}=1.08 \mathrm{mg} / \mathrm{s}\right), \phi=90 \mathrm{deg}$. 
effects on ions and neutrals. Little is known about highenergy ion generation in this environment.

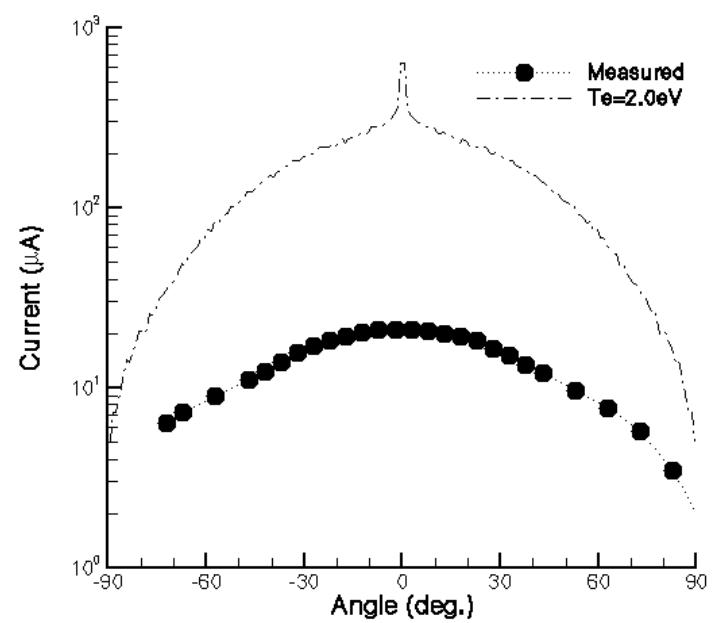

Figure 18. Angular ion emission profiles at the RPA position for case $10\left(\mathrm{I}_{\mathrm{ck}}=20.0 \mathrm{~A}, \mathrm{~F}=1.08 \mathrm{mg} / \mathrm{s}\right)$.

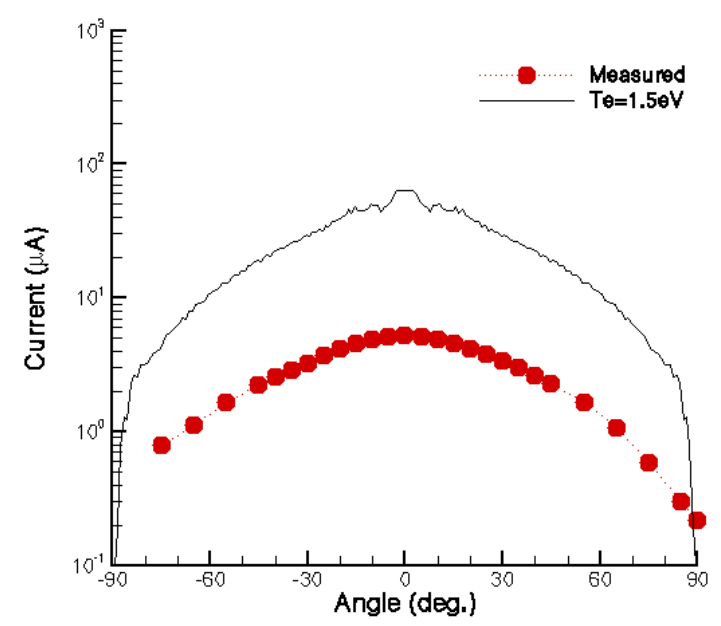

Figure 19. Angular ion emission profiles at the RPA position for case $4\left(\mathrm{I}_{\mathrm{ck}}=4.0 \mathrm{~A}, \mathrm{~F}=0.50 \mathrm{mg} / \mathrm{s}\right)$.

\section{CONCLUDING REMARKS}

A characterization study of the T6 hollow cathode has been performed. Far-field energy, angular, flux, and charge state data have been obtained for emitted ions. Numerous operating points of the T6 were studied, revealing some with unacceptably high ion energy for use inside a thruster and others that are more benign. The ion energy distribution functions were emphasized in this study. These functions were generally simpler and less energetic than those observed in other cathodes previously.

Simulation with combined PIC and DSMC methods has produced results in excellent agreement with the measured ion energy distribution functions under a variety of conditions, using only electron temperature as an adjustable parameter. Significant discrepancies appear in the comparisons of angular distributions of ion emission, and in the potential and electron temperature gradients in the restricted plume region that was investigated. Because retarding potential profiles exhibit little dependence on charge state and electron temperature has been the most important parameter in reproducing the T5 and T6 ion energy distribution functions, it is likely that a potential hill acceleration mechanism predominantly causes ion acceleration. The influence of other parameters, particularly the ion-electron collision frequency in T5 simulations, may indicate that the mechanism is not purely potential hill. The present study did not explore the very high ion energy regime that has been observed for specific cathodes when mounted in simulated ion thruster discharge chambers, a more complex situation.

These results carry implications for vacuum arc plasmas, where similar energy distributions are obtained from a discharge of about the same $\Delta \mathrm{V}$, where debate has persisted for decades concerning the ion acceleration mechanism.

Table 1. Selected operating point data.

\begin{tabular}{ccccc}
\hline \hline $\begin{array}{c}\text { Operating } \\
\text { Point }\end{array}$ & $\begin{array}{c}\text { Keeper } \\
\text { Current, } \mathrm{I}_{\mathrm{ck}}\end{array}$ & $\begin{array}{c}\text { Keeper } \\
\text { Voltage, } \mathrm{V}_{\mathrm{ck}}\end{array}$ & $\begin{array}{c}\text { Flow Rate, } \\
\mathrm{F}(\mathrm{mg} / \mathrm{s})\end{array}$ & $\begin{array}{c}\text { Test Chamber } \\
\text { Pressure (Torr) }\end{array}$ \\
\hline 1 & 1.5 & 28 & 0.12 & $1.7 \times 10^{-5}$ \\
2 & 4.0 & 26.3 & 0.125 & $\approx 2.0 \times 10^{-5}$ \\
3 & 8.0 & 26 & 0.125 & $1.3-3.2 \times 10^{-5}$ \\
4 & 4.0 & 20.4 & 0.50 & $5.9 \times 10^{-5}$ \\
5 & 8.0 & 20.2 & 0.50 & $7.8 \times 10^{-5}$ \\
6 & 12.0 & 20.8 & 0.50 & $\approx 1.2 \times 10^{-4}$ \\
7 & 4.0 & 10.4 & 1.08 & $1.1 \times 10^{-4}$ \\
8 & 8.0 & 11.0 & 1.08 & $1.2 \times 10^{-4}$ \\
9 & 12.0 & 11.4 & 1.08 & $1.2 \times 10^{-4}$ \\
10 & 20.0 & 14.0 & 1.08 & $\approx 1.5 \times 10^{-4}$ \\
\hline \hline
\end{tabular}




\section{Acknowledgments}

The preparation of this manuscript was supported by The Aerospace Corporation through its IRAD Program. IDB gratefully acknowledges Dr. Matt Domonkos of NASA Glenn Research Center for use of his hollow cathode analysis codes. The hollow cathode was provided by D.G. Fearn and N.C. Wallace of the Defence Evaluation and Research Agency (now Qinetic).

\section{$\underline{\text { References }}$}

1. Kameyama, I., and Wilbur, P.J., "Zenith-Angle Distributions of Erosion Rates near High-Current Hollow Cathodes," AIAA Paper 96-3208, July 1996.

2. Latham, P.M., Pearce, A.J., and Bond, R.A., "Erosion Processes in the UK-25 Ion Thruster," IEPC Paper 91096, Oct. 1991

3. Friedly, V.J., and Wilbur, P.J., "High Current Hollow Cathode Phenomena," J. Propulsion and Power, Vol. 8 (3), 1992, pp. 635-643.

4. Kameyama, I., and Wilbur, P.J., "Measurements of Ions from High-Current Hollow Cathodes Using Electrostatic Energy Analyzer," J. Propulsion and Power, Vol. 16, No. 3, 2000, pp. 529-535.

5. Crofton, M.W., "Preliminary Mass Spectroscopy of a Xenon Hollow Cathode," J. Propulsion and Power, Vol. 16 , No. 1, 2000, pp. 157-159.

6. Kameyama, I., and Wilbur, P., "Potential-Hill Model of High Energy Ion Production near High-Current Hollow Cathodes," ISTS Paper 98-a-2-17, 21st International Symposium on Space Technology and Science, Omiya, May 1998.

7. Patterson, S.W., and Fearn, D.G., "The Generation of High Energy Ions in Hollow Cathode Discharges," IEPC Paper 99-125, Oct. 1999.

8. Yushkov, G.Y., Anders, A., Oks, E.M., and Brown, I.G., "Ion Velocities in Vacuum Arc Plasmas," J. Applied Physics, Vol. 88, No. 10, 2000, pp. 5618-5622.

9. Wieckert, C., "The Expansion of the Cathode Spot Plasma in Vacuum Arc Discharges," Physics of Fluids, Vol. 30, No. 6, 1987, pp. 1810-183.

10. Williams, G.J. Jr., Smith, T.B., Domonkos, M.T., Gallimore, A.D., and Drake, R.P., "Laser Induced Fluorescence Characterization of Ions Emitted from Hollow Cathodes," IEEE Transactions on Plasma Science, Vol. 28, No. 5, 2000, pp. 1664-1675.

11. Crofton, M.W., and Boyd, I.D., "Plume Measurement and Modeling Results for a Xenon Hollow Cathode," AIAA Paper 2002-4103, July 2002.

12. Boyd, I.D., and Crofton, M.W., "Modeling the Plasma Plume of a Hollow Cathode," to be published in J. Appl. Phys., 2003.
13. Crofton, M.W., "Evaluation of the United Kingdom Ion Thruster," J. Spacecraft and Rockets, Vol. 33, No. 5, 1996, pp. 739-747, and references therein.

14. Simpson, H.B., Wallace, N.C., Fearn, D.G., and Kelly, M.K., "A Summary of the Qinetiq Hollow Cathode Development Programme in Support of European High Power Hall Effect and Gridded Thrusters," IEPC Paper 03-0214, March 2003.

15. Farnell, C.C., Williams, J.D., and Wilbur, P.J., "Characteristics of Energetic Ions Emitted from Hollow Cathodes," IEPC Paper 03-072, March 2003.

16. Parks, D. E., Mandell, M. J., and Katz, I., "Fluid Model of Plasma Outside a Hollow Cathode Neutralizer," Journal of Spacecraft and Rockets, Vol. 19, 1982, pp. 354-357.

17. Williams, J. D. and Wilbur, P. J., "Electron Emission from a Hollow Cathode-Based Plasma Contactor," Journal of Spacecraft and Rockets, Vol. 29, 1992, pp. 820-829.

18. Birdsall, C. K. and Langdon, A. B., Plasma Physics Via Computer Simulation, Adam Hilger Press, 1991.

19. Bird, G. A., Molecular Gas Dynamics and the Direct Simulation of Gas Flows, Oxford University Press, 1994.

20. Mitchner, M. and Kruger, C. H., Partially Ionized Gases, Wiley, New York, 1973.

21. Domonkos, M. T., "Evaluation of Low-Current Orificed Hollow Cathodes," Doctoral Thesis, Department of Aerospace Engineering, University of Michigan, September 1999.

22. Crawford, F.T. and Gabriel, S.B., "Numerical Simulation of the Hollow Cathode Plasma using a PIC-DSMC Code," IEPC Paper 03-27, March 2003.

23. Miller, D.R., "Free Jet Sources," Atomic and Molecular Beam Methods, edited by G. Scoles, Vol. 1, Oxford, New York, 1988, pp. 14-53.

24. Von Engel, A., Ionized Gases, Oxford, London, 1965.

25. Davis, W.D., and Miller, H.C., "Analysis of the Electrode Products Emitted by dc Arcs in a Vacuum Ambient," Journal of Applied Physics, Vol. 40, No. 5, 1969, pp. 2212-2221.

26. Tsuruta, K., Sekiya, K., and Watanabe, G., "Velocities of Copper and Silver Ions Generated from an Impulse Vacuum Arc," IEEE Transactions on Plasma Science, Vol. 25, No. 4, 1997, pp. 603-608. 